\title{
WEIGHTED POWER MEAN DISCRETE DYNAMICAL SYSTEMS: FAST CONVERGENCE PROPERTIES
}

\author{
FRANCISCO J. SOLIS
}

Received 9 February 2006; Accepted 25 April 2006

We studied families of discrete dynamical systems obtained by using iteration functions given by weighted power mean in order to understand the role of hyperrapid convergence in nonlinear maps. Our interest resides in concepts related to the velocity of convergence. We introduce new concepts regarding the time of convergence and we provide an ordering of these families according to their dependence on parameters.

Copyright (c) 2006 Francisco J. Solis. This is an open access article distributed under the Creative Commons Attribution License, which permits unrestricted use, distribution, and reproduction in any medium, provided the original work is properly cited.

\section{Introduction}

The arithmetico-geometrical algorithm is well known for the numerical evaluation of elliptic functions and integrals [8]. The algorithm starts with two numbers $\left(x_{0}, y_{0}\right)$ and successive numbers $\left(x_{n}, y_{n}\right), n \in \mathbb{N}$, are calculated from the recurrence formulas $x_{n+1}=$ $\left(x_{n}+y_{n}\right) / 2$ (arithmetic mean) and $y_{n+1}=\sqrt{x_{n} y_{n}}$ (geometric mean). Thus, a convergent sequence $\left\{\left(x_{n}, y_{n}\right)\right\}$ is generated with a common limit given by an elliptic integral.

The joint iteration of the arithmetic and the geometric means has been explored already by Carl Friedreich Gauss in a related problem regarding secular perturbations of orbital elements [5]. The algorithm has found many uses in several disciplines, in areas of mathematics such as numerical analysis $[2,3]$, number theory $[1,4]$, in physics [7, 9], in finances in problems related to portfolio market value [6], and so on. In spite of the applicability of this process, there has not been much attention devoted to dynamical processes with different versions of iterated means. In this work we will introduce and explore new dynamical systems with an iteration function given by a generalization of the arithmetic and geometric means. Our interest resides in the study of some dynamical properties such as convergence and velocity. We establish a classification on these new dynamical systems according to the time of convergence and the critical exponent associated concepts that will be introduced afterwards. 
There is a huge variety of possible generalizations of the arithmetic and geometric means. One of them is given by a weighted power mean, which is defined as follows: given two positive numbers $x$ and $y$ and a weight $w$, with $0<w<1$, their weighted power mean is defined as $\left(w x^{r}+(1-w) y^{r}\right)^{1 / r}$, where the power $r$ is a nonzero real number. We are in position to state the next definition.

Definition 1.1. A weighted power mean function is defined as the function $f: R^{+} \times R^{+} \times$ $(0,1) \times R^{+} \rightarrow R$ with

$$
f(x, y, w, r)=\left(w x^{r}+(1-w) y^{r}\right)^{1 / r}
$$

It is straightforward to show that a power mean function $f$ satisfies the following properties.

(1)

$$
\lim _{r \rightarrow 0} f(x, y, w, r)=x^{w} y^{1-w}
$$

So we defined $f(x, y, w, 0)=x^{w} y^{1-w}$. Notice that in particular when $w=1 / 2$ we obtain the geometric mean of $x$ and $y$, that is $f(x, y, 1 / 2,0)=\sqrt{x y}$.

(2) $f(x, y, w, r)$ is an increasing function of $r$ for each fixed $w, x$, and $y$.

(3) If $x \leq y$, then $f(x, y, w, r)$ is an increasing (decreasing) function of $w$ for each fixed $x, y$, and $r>0,(r<0)$.

(4) If $x \leq y$, then the harmonic weighted power mean, $f(x, y, w,-1)$, is related to the arithmetic, $f(x, y, w, 1)$, and geometric, $f(x, y, w, 0)$, weighted power mean as

$$
x \leq f(x, y, w,-1) \leq f(x, y, w, 0) \leq f(x, y, w, 1) \leq y .
$$

This paper is organized as follows. In Section 2 we define and give properties of weighted power mean discrete dynamical systems. In Section 3 the dynamic behavior of the dynamical systems is analyzed by reducing their dimension. In Section 4 we introduce the concepts of convergence and critical exponents in a general setting. Numerical experiments between the different systems and conclusions are given in Section 5.

\section{Weighted power mean systems}

Let us start with the following definition.

Definition 2.1. Given a weighted power mean function $f$ with fixed $w_{i}$ and $r_{i}, i=1,2$, a weighted power mean (WPM) discrete dynamical system is defined as

$$
\begin{aligned}
& x_{n+1}=f\left(x_{n}, y_{n}, w_{1}, r_{1}\right), \\
& y_{n+1}=f\left(x_{n}, y_{n}, w_{2}, r_{2}\right),
\end{aligned}
$$

with $0<x_{0}$ and $0<y_{0}$. 
In the successive sections we study some elementary properties of WPM discrete dynamical systems.

\subsection{Convergence}

Proposition 2.2. The WPM discrete dynamical system (2.1) converges for appropriate initial conditions to a common value.

Proof. Let $x_{0}$ and $y_{0}$ be nonnegative initial conditions for the system (2.1), without loss in generality we assume that $x_{0} \leq y_{0}$, then we have that $x_{0}^{r} \leq w_{1} x_{0}^{r}+\left(1-w_{1}\right) y_{0}^{r}$. Thus,

$$
x_{0} \leq x_{1}=f\left(x_{0}, y_{0}, w_{1}, r_{1}\right) \leq y_{0}
$$

inductively we obtain that the sequence $\left\{x_{n}\right\}_{0}^{\infty}$ satisfies

$$
x_{0} \leq x_{1} \leq \cdots \leq x_{n} \leq y_{0}, \quad n \in \mathbb{N}
$$

and similarly

$$
x_{0} \leq y_{n} \leq \cdots \leq y_{1} \leq y_{0}, \quad n \in \mathbb{N} \text {. }
$$

Thus $\left\{x_{n}\right\}_{0}^{\infty}$ and $\left\{y_{n}\right\}_{0}^{\infty}$ are two convergent sequences. Let $x_{\infty}$ and $y_{\infty}$ be their corresponding limits. Using (2.1) we get that $x_{\infty}^{r_{i}}=y_{\infty}^{r_{i}}$ for $i=1,2$, which implies that $x_{\infty}=y_{\infty}$.

The limit values of WPM discrete dynamical systems can be calculated explicitly for several specific cases which we study in the following sections.

2.2. Case $r_{1}=r_{2}$ (linear). Let us consider the case where $r_{1}$ and $r_{2}$ have the same value which we denote as $r$.

Proposition 2.3. The WPM discrete dynamical system

$$
\begin{aligned}
& x_{n+1}=\left(w_{1} x_{n}^{r}+\left(1-w_{1}\right) y_{n}^{r}\right)^{1 / r}, \\
& y_{n+1}=\left(w_{2} x_{n}^{r}+\left(1-w_{2}\right) y_{n}^{r}\right)^{1 / r}
\end{aligned}
$$

converges to a common value given by

$$
x_{\infty}=y_{\infty}=\frac{w_{2} x_{0}^{r}+\left(1-w_{1}\right) y_{0}^{r}}{1-w_{1}+w_{2}} .
$$

Proof. In this case the discrete dynamical system (2.5) can be written as

$$
\begin{aligned}
& x_{n+1}^{r}=w_{1} x_{n}^{r}+\left(1-w_{1}\right) y_{n}^{r}, \\
& y_{n+1}^{r}=w_{2} x_{n}^{r}+\left(1-w_{2}\right) y_{n}^{r} .
\end{aligned}
$$


4 Weighted power mean discrete dynamical systems

Setting $\hat{x}_{n}=x_{n}^{r}$ and $\hat{y}_{n}=y_{n}^{r}$ in (2.7) we get the linear system

$$
\begin{aligned}
& \hat{x}_{n+1}=w_{1} \hat{x}_{n}+\left(1-w_{1}\right) \hat{y}_{n}, \\
& \hat{y}_{n+1}=w_{2} \hat{x}_{n}+\left(1-w_{2}\right) \hat{y}_{n} .
\end{aligned}
$$

The linear system in $\hat{x}_{n}$ and $\hat{y}_{n}$ can be written in the form

$$
X_{n+1}=A X_{n}
$$

with

$$
X_{n}=\left(\begin{array}{l}
\hat{x}_{n} \\
\hat{y}_{n}
\end{array}\right), \quad A=\left(\begin{array}{ll}
w_{1} & 1-w_{1} \\
w_{2} & 1-w_{2}
\end{array}\right) .
$$

Thus

$$
\lim _{n \rightarrow \infty} X_{n}=\lim _{n \rightarrow \infty} A^{n} X_{0}=\left(\begin{array}{ll}
w_{2} & 1-w_{1} \\
w_{2} & 1-w_{1}
\end{array}\right)\left(\begin{array}{l}
\hat{x}_{0} \\
\hat{y}_{0}
\end{array}\right) \frac{1}{1-w_{1}+w_{2}} .
$$

Notice that $\hat{x}_{n}$ and $\hat{y}_{n}$ converge to the common limit given by

$$
x_{\infty}=y_{\infty}=\frac{w_{2} \hat{x}_{0}+\left(1-w_{1}\right) \hat{y}_{0}}{1-w_{1}+w_{2}} .
$$

Therefore, the common limit of the original system (2.5) is given by

$$
x_{\infty}=y_{\infty}=\frac{w_{2} x_{0}^{r}+\left(1-w_{1}\right) y_{0}^{r}}{1-w_{1}+w_{2}} .
$$

Notice that the system converges exponentially, that is, as $\exp \left(n \ln \left(\left|w_{1}-w_{2}\right|\right)\right)$ with $n$ approaching $\infty$.

2.3. Case with $w_{1}=w_{2}=1 / 2$ and $r_{2}=0$

Proposition 2.4. For given initial conditions the discrete dynamical system

$$
\begin{aligned}
& x_{n+1}=\left(\frac{x_{n}^{r}+y_{n}^{r}}{2}\right)^{1 / r}, \\
& y_{n+1}=\sqrt{x_{n} y_{n}}
\end{aligned}
$$

converges to the common value $L$ which satisfies

$$
L=\frac{\pi}{2}\left(\int_{0}^{\pi / 2} \frac{d \theta}{\sqrt{x_{0}^{2 r} \cos ^{2}(\theta)+y_{0}^{2 r} \sin ^{2}(\theta)}}\right)^{-1}
$$

Proof. Let us substitute

$$
k_{n}=\frac{x_{n}^{r}-y_{n}^{r}}{x_{n}^{r}+y_{n}^{r}}
$$


into the following equality known as Gauss's transformation:

$$
\frac{1}{1+k_{n}} \int_{0}^{\pi / 2} \frac{d \theta}{\sqrt{1-4 k_{n} \sin ^{2}(\theta) /\left(1+k_{n}\right)^{2}}}=\int_{0}^{\pi / 2} \frac{d \theta}{\sqrt{1-k_{n}^{2} \sin ^{2}(\theta)}}
$$

to get

$$
\int_{0}^{\pi / 2} \frac{d \theta}{\sqrt{x_{n}^{2 r} \cos ^{2}(\theta)+y_{n}^{2 r} \sin ^{2}(\theta)}}=\int_{0}^{\pi / 2} \frac{d \theta}{\sqrt{x_{0}^{2 r} \cos ^{2}(\theta)+y_{0}^{2 r} \sin ^{2}(\theta)}}
$$

Taking the limit as $n$ goes to infinity and using the fact that the system (2.14) has a common limit we obtain the desired result.

This system converges faster than the system with $r_{1}=r_{2}$. Later on we will show in detail the reason for this behavior.

\section{Decoupling}

Using the following transformation $x_{n}=\rho_{n} \cos \left(\theta_{n}\right)$ and $y_{n}=\rho_{n} \sin \left(\theta_{n}\right)$ with $0<\theta_{n}<\pi / 2$ for all $n$, the system (2.1) decouples into

$$
\begin{gathered}
\tan \theta_{n+1}=\frac{\left(w_{1}+\left(1-w_{1}\right) \tan ^{r_{1}}\left(\theta_{n}\right)\right)^{1 / r_{1}}}{\left(w_{2}+\left(1-w_{2}\right) \tan ^{r_{2}}\left(\theta_{n}\right)\right)^{1 / r_{2}}} \\
\rho_{n+1}=\rho_{n} \cos \left(\theta_{n}\right) G\left(\theta_{n}\right)
\end{gathered}
$$

where

$$
G\left(\theta_{n}\right)=\sqrt{\left(w_{1}+\left(1-w_{1}\right) \tan ^{r_{1}}\left(\theta_{n}\right)\right)^{2 / r_{1}}+\left(w_{2}+\left(1-w_{2}\right) \tan ^{r_{2}}\left(\theta_{n}\right)\right)^{2 / r_{2}}} .
$$

Writing $z_{n}=\tan \left(\theta_{n}\right)$ in (3.1), we obtain a one-dimensional discrete system $z_{n+1}=$ $H\left(z_{n}\right)$ given by

$$
z_{n+1}=\frac{\left(w_{1}+\left(1-w_{1}\right) z_{n}^{r_{1}}\right)^{1 / r_{1}}}{\left(w_{2}+\left(1-w_{2}\right) z_{n}^{r_{2}}\right)^{1 / r_{2}}}=H\left(z_{n}\right)
$$

This system inherits the convergence properties of system (2.1), therefore it converges globally to the fixed point $z=1$ for all values of $w_{i}$ and $r_{i}, i=1,2$. Notice that $\left|H^{\prime}(1)\right|=$ $\left|w_{1}-w_{2}\right|<1$ which implies that if $w_{1} \neq w_{2}$, then the system converges exponentially to the fixed point and the error decays as $e^{-n / \tau}$ where $\tau$ is a constant, see [10]. 
Assume now that $w_{1}=w_{2}$, then $\left|H^{\prime}(1)\right|=0$ and $\left|H^{\prime \prime}(1)\right|=w(1-w)\left|r_{1}-r_{2}\right|$. Notice that $H^{\prime \prime}(1)=0$ only if $r_{1}=r_{2}$ and this is the case which was already analyzed in Section 2.2. Therefore we can assume that $H^{\prime \prime}(1) \neq 0$. Let us now investigate this case in the next section using a general setting.

\section{Critical exponents and time of convergence}

Given a discrete dynamical system of the form $x_{n+1}=H\left(\lambda, x_{n}\right)$, where $\lambda$ is a parameter, assume the existence of an isolated attracting fixed point $x_{H}(\lambda)$, which may depend on $\lambda$. Define the error sequence, $\left\{\epsilon_{n}\right\}$, as $x_{n}=x_{H}+\epsilon_{n}$. Therefore

$$
\epsilon_{n+1}=H^{\prime}\left(\lambda, x_{H}\right) \epsilon_{n}+H^{\prime \prime}\left(\lambda, x_{H}\right) \frac{\epsilon_{n}^{2}}{2}+O\left(\epsilon_{n}^{3}\right)
$$

Let $\hat{\lambda}$ be a value of the control parameter satisfying $H^{\prime}\left(\hat{\lambda}, x_{H}\right)=0$, and assume that $H^{\prime \prime}\left(\hat{\lambda}, x_{H}\right)$ is not identically equal to zero, then using (4.1) we conclude that $\left|\epsilon_{n}\right|$ decays as $\exp \left(-2^{n} / \tau\right)$ with $\tau$ a constant independent of $n$. From now on we will refer to the points $\left(\hat{\lambda}, x_{H}\right)$ as points of fast convergence. The constant $\tau$ has a particular meaning, which is given in the next definition.

Definition 4.1. Define the time of convergence of the system $x_{n+1}=H\left(\lambda, x_{n}\right)$ at a point of fast convergence $\left(\hat{\lambda}, x_{H}\right)$ as

$$
\tau=-\left(\ln \left|\frac{\epsilon_{0}}{2} \frac{\partial^{2} H}{\partial x^{2}}\left(\hat{\lambda}, x_{H}\right)\right|\right)^{-1}
$$

and we also define the critical exponent, $\delta$, as the smallest power of the nonzero term in the Taylor series of $g(\lambda)=\ln \left|\left(\epsilon_{0} / 2\right)\left(\partial^{2} H / \partial x^{2}\right)\left(\lambda, x_{H}\right)\right|$ around the point $\hat{\lambda}$.

It is noticeable that both concepts depend on the initial condition. With these definitions, we obtain a classification of discrete dynamical systems at points of fast convergence. For each class, defined by specific values of $\lambda$ and derivatives of the function $\ln \left|\left(\epsilon_{0} / 2\right)\left(\partial H^{2} / \partial x^{2}\right)\left(\lambda, x_{H}\right)\right|$, the value of $\delta$ is independent of the iteration function. The main ideas to define these new concepts are taken from [10] which is a work regarding slower dynamical systems.

We now return to the analysis of WPM dynamical systems to show the existence of classes of dynamical systems with different associated critical exponent values.

For the system

$$
z_{n+1}=\frac{\left(w+(1-w) z_{n}^{r_{1}}\right)^{1 / r_{1}}}{\left(w+(1-w) z_{n}^{r_{2}}\right)^{1 / r_{2}}}
$$

$(w, 1)$ is a point of fast convergence for all $w \in(0,1)$. So considering a fixed weight $w_{0}$, the Taylor expansion of the function $g(w)=\ln \left(\left(\epsilon_{0} / 2\right) w(1-w)\left|r_{1}-r_{2}\right|\right)$ around the value $w_{0}$ 


$$
\begin{aligned}
g(w)= & \ln \left(\frac{\epsilon_{0}}{2} w_{0}\left(1-w_{0}\right)\left|r_{1}-r_{2}\right|\right)-\frac{2 w_{0}-1}{w_{0}\left(w_{0}-1\right)}\left(w-w_{0}\right) \\
& +\left(\frac{2}{w_{0}\left(1-w_{0}\right)}-\frac{2 w_{0}-1}{w_{0}\left(1-w_{0}\right)^{2}}-\frac{2 w_{0}-1}{w_{0}^{2}\left(1-w_{0}\right)}\right) \frac{\left(w-w_{0}\right)^{2}}{2}+O\left(\left(w-w_{0}\right)^{3}\right) .
\end{aligned}
$$

Therefore we obtain that if $\epsilon_{0} w_{0}\left(1-w_{0}\right)\left|r_{1}-r_{2}\right| \neq 2$, the system (4.3) has associated a critical exponent $\delta=0$. So except for a set of Lebesgue measure zero, that is, when $\epsilon_{0} w_{0}\left(1-w_{0}\right)\left|r_{1}-r_{2}\right| \neq 2$, zero is the typical value of the critical exponent for WPM systems with the same weight.

Now assume that $\epsilon_{0} w_{0}\left(1-w_{0}\right)\left|r_{1}-r_{2}\right|=2$, requiring that $\epsilon_{0}\left|r_{1}-r_{2}\right| \geq 8$, then the system (4.3) has a critical exponent $\delta=1$ only if $w_{0} \neq 1 / 2$. Finally, the only existing critical exponent is $\delta=2$ if we have that $w_{0}=1 / 2$ and $\epsilon_{0}\left|r_{1}-r_{2}\right|=8$. Therefore we have proven the following proposition.

Proposition 4.2. The discrete dynamical system

$$
z_{n+1}=\frac{\left(w+(1-w) z_{n}^{r_{1}}\right)^{1 / r_{1}}}{\left(w+(1-w) z_{n}^{r_{2}}\right)^{1 / r_{2}}}
$$

has three associated critical exponents at the fast convergence point $\left(w_{0}, 1\right)$ with $w_{0} \in(0,1)$. The value $\delta=0$ corresponds to the case where $\left(z_{0}-1\right) w_{0}\left(1-w_{0}\right)\left|r_{1}-r_{2}\right| \neq 2$. The value $\delta=1$ is possible if and only if $\left(z_{0}-1\right) w_{0}\left(1-w_{0}\right)\left|r_{1}-r_{2}\right|=12$ and $w_{0} \neq 1 / 2$, and $\delta=2$ for the cases where $w_{0}=1 / 2$ and $\left(z_{0}-1\right)\left|r_{1}-r_{2}\right|=8$.

\section{Numerical examples and conclusions}

The WPM discrete dynamical system

$$
\begin{aligned}
& x_{n+1}=\left(w_{1} x_{n}^{r_{1}}+\left(1-w_{1}\right) y_{n}^{r_{1}}\right)^{1 / r_{1}}, \\
& y_{n+1}=\left(w_{2} x_{n}^{r_{2}}+\left(1-w_{2}\right) y_{n}^{r_{1} 2}\right)^{1 / r_{2}}
\end{aligned}
$$

(1) converges exponentially if $w_{1} \neq w_{2}$. In Figure 5.1 we show the set of initial conditions $\left\{\left(x_{0}, y_{0}\right) \mid 0 \leq x_{0} \leq 1,0 \leq y_{0} \leq 1\right\}$ for a WPM system with $r_{1}=2, r_{2}=4$, $w_{1}=0.5$, and $w_{2}=0.3$. The number of iterations necessary to achieve convergence with a tolerance of $10^{-6}$ is shown in the different colored regions of the unit square. The black portion of Figure 5.1 means that only one iteration is needed to achieve convergence; 


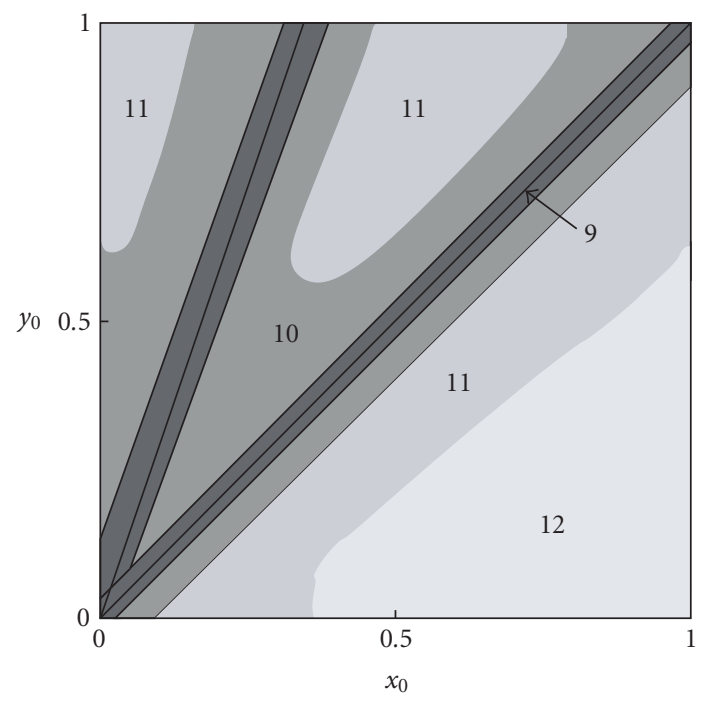

Figure 5.1. Number of iterations with exponential decay.



Figure 5.2. Number of iterations with critical exponent $\delta=0$.

(2) with $w_{1}=w_{2}$, converges with a critical exponent of $\delta=0$ for all initial conditions except for a set of measure zero. In Figure 5.2, we show the number of iterations to achieve convergence with a tolerance of $10^{-6}$ in the unit square of initial conditions for a system with $r_{1}=5, r_{2}=0.5, w_{1}=w_{2}=0.3$. Notice the notable reduction in the number of iterations from the previous example. 


\section{References}

[1] J. M. Borwein and P. B. Borwein, The arithmetic-geometric mean and fast computation of elementary functions, SIAM Review 26 (1984), no. 3, 351-366.

[2] R. P. Brent, Fast multiple-precision evaluation of elementary functions, Journal of the Association for Computing Machinery 23 (1976), no. 2, 242-251.

[3] B. C. Carlson, Algorithms involving arithmetic and geometric means, The American Mathematical Monthly 78 (1971), no. 5, 496-505.

[4] P. D. T. A. Elliott, Arithmetic Functions and Integer Products, Grundlehren der Mathematischen Wissenschaften, vol. 272, Springer, New York, 1985.

[5] C. F. Gauss, Determinatio attractionis, quam in punctum quodvis positionis datae exerceret planetam si eius massa per totam orbita, ratione temporis, quo singulae partes describuntur, uniformiter esset dispertita. Theorematis fundamentalis in doctrina residorum qaudraticis demonstrationes et ampliationes novae, Commentationes Societatis Regiae Scientiarum Gotti 4 (1818), 21.

[6] H. M. Hulburt and K. V. Chow, Value, size, and portfolio efficiency, Journal of Portfolio Management 26 (2000), no. 3, 78-89.

[7] O. D. Kellogg, Foundations of Potential Theory, Die Grundlehren der Mathematischen Wissenschaften, vol. 31, Springer, Berlin, 1967.

[8] L. V. King, On the Direct Numerical Calculation of Elliptic Functions and Integrals, Cambridge University Press, Cambridge, 1924.

[9] M. Pick, J. Picha, and V. Vyskocil, Theory of the Earth's Gravity Field, Academia, Prague, 1973.

[10] F. J. Solis and R. Felipe, Slow convergence of maps, Nonlinear Studies 8 (2001), no. 3, 389-394.

Francisco J. Solis: Departament de Matemáticas Aplicadas, Centro de Investigación en Matemáticas, Callejon Jalisco s/n, Valenciana, Guanajuato, Gto., CP 36000, Mexico

E-mail address: solis@cimat.mx 


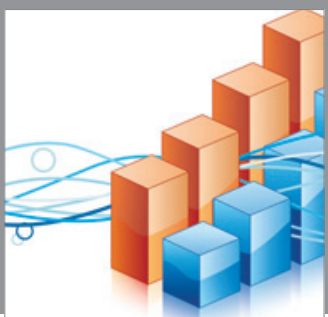

Advances in

Operations Research

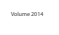

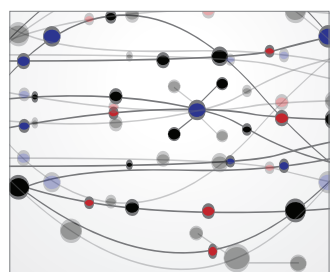

\section{The Scientific} World Journal
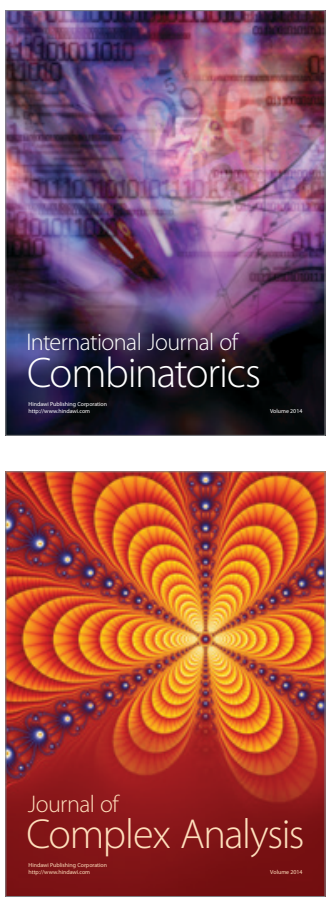

International Journal of

Mathematics and

Mathematical

Sciences
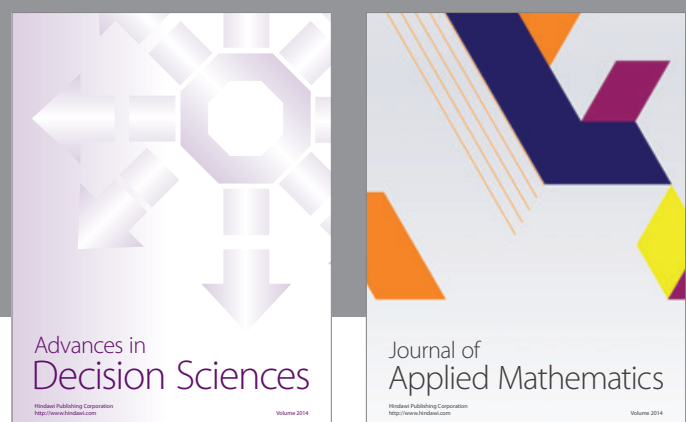

Journal of

Applied Mathematics


Submit your manuscripts at http://www.hindawi.com
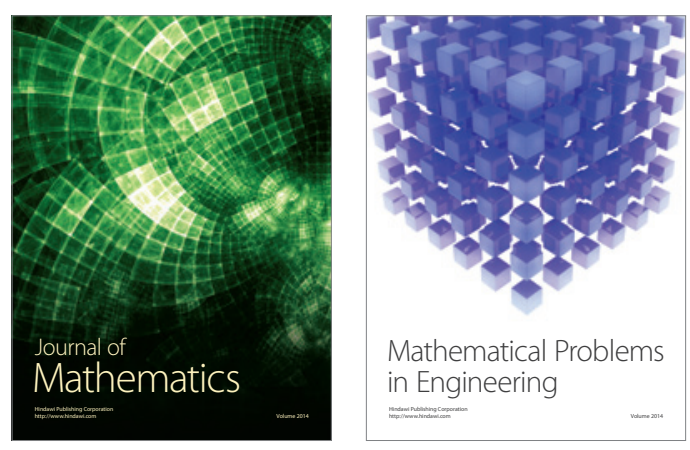

Mathematical Problems in Engineering
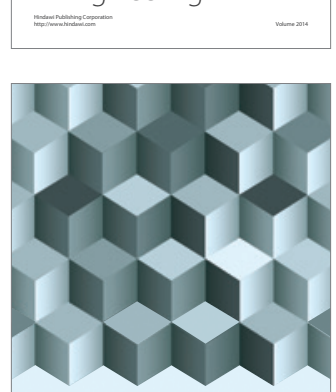

Journal of

Function Spaces
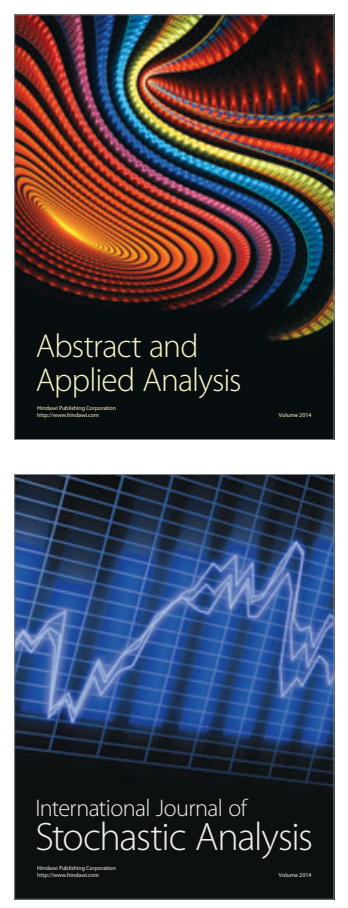



ournal of

Probability and Statistics

Promensencen
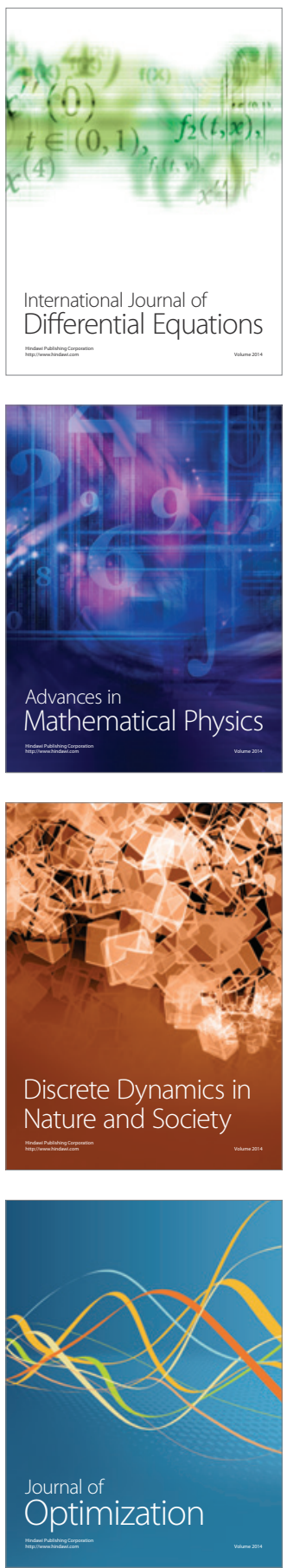